\title{
Editorial
}

\section{Antioxidanzien in Prävention und Therapie - wo stehen wir?}

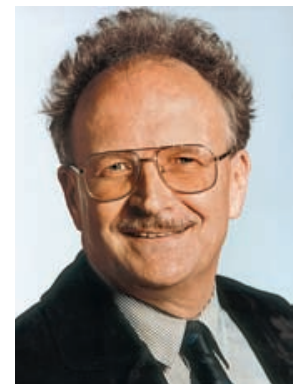

Linus Pauling hatte schon in den 30er-Jahren des letzten Jahrhunderts aufgrund quantenmechanischer Überlegungen die Existenz des Superoxid-Radikals - eines hochreaktiven Reduktionsproduktes des molekularen Sauerstoffs - postuliert und nach der Arbeit von Denham Harmann (1956) über die Bedeutung von Sauerstoffradikalen für den Alterungsprozess wurden seit den 60er-Jahren zunehmend die experimentellen Grundlagen über die physiologische und pathologische Bedeutung aktivierter Formen des Sauerstoffs gelegt.

Mit der Monografie „Antioxidant Adaptation“ von Steven Levine und Paris Kidd (1985) setzte eine stürmische Entwicklung ein und der „oxidative Stress“ wurde zu einer Metapher für ein universelles pathogenetisches Prinzip, das als kausales Erklärungsmodell für eine Vielzahl von Erkrankungen bis hin zur Alterung herangezogen wurde. Diesem Megatrend entsprechend schossen mehr oder weniger wissenschaftliche nationale und internationale Gesellschaften und informelle Clubs aus dem Boden.

Auf dieses eingängige Erklärungsmodell wurden dann rasch präventive und therapeutische Interventionen aufgesetzt und es kam zur Entwicklung einer inzwischen unüberschaubaren Zahl verschiedenster Produkte im Bereich von Nahrungsergänzungen, Kosmetika, ergänzenden bilanzierten Diäten, Medizinprodukten, Arzneimitteln, ja selbst Textilien mit der Auslobung eines Zellschutzes durch antioxidative Wirkung.

Nach Durchführung zahlreicher groß angelegter epidemiologischer Untersuchungen und Interventionsstudien zeigte sich allerdings, dass sich die physiologischen Redox-Regulationsmechanismen in Zellen und Geweben komplexer darstellen als sie im Design der meisten Studien berücksichtigt wurden. So konnte es beispielsweise bei Einsatz höherer Dosierungen einzelner oder weniger Antioxidanzien zu Ergebnissen kommen, die dann als paradox interpretiert werden mussten (z.B. „The Ascorbic Acid Paradox“, Biochem Biophys Res Commun 2010; 400: 466-470), da völlig inadäquate und irrelevante Studiendesigns vorlagen. So war nach derartigen Studien Vitamin C plötzlich genotoxisch. Aus diesen Gründen sind die Ergebnisse vieler teilweise sehr teurer Studien und ganz besonders die Ergebnisse der daraus abgeleiteten Metaanalysen (z.B. Cochrane etc.) als wertlos, ja irreführend einzustufen.

In den Medien ist diese unbefriedigende und teilweise kontroverse Situation begierig aufgegriffen worden, hat zu einer Polarisierung der Expertenmeinungen von glühenden Anhängern bis zu fanatischen Gegnern geführt und Schlagzeilen wie „Vitaminlüge“ etc. produziert. Als Resultat ist eine Verunsicherung der Bevölkerung zu konstatieren und ein Verlust an Glaubwürdigkeit der Experten, wie man dies sonst nur von der Politik kennt.

Dass es auch anders gehen kann, zeigt eine im Rahmen des britischen National
Diet and Nutrition Survey durchgeführte Studie, deren Ergebnisse ganz aktuell von Bates et al. publiziert wurden (British Journal of Nutrition 2011; 105: 123-132). Hierbei wurde zwischen 1994 und 2008 die Mortalität von ursprünglich 1054 Studienteilnehmern mit einem mittleren Lebensalter von 76,6 Jahren erfasst, wobei Frauen und Männer gleich verteilt waren. 74\% der Männer und 62\% der Frauen verstarben im Untersuchungszeitraum. Der Studienendpunkt Mortalität gilt als das aussagekräftigste Kriterium für eine abschließende Bewertung.

Sowohl die Plasmaspiegel als auch die Aufnahmemengen Redox-modulierender Substanzen zu Beginn der Studie erwiesen sich als signifikante Prädiktoren des Mortalitätsrisikos - unabhängig von den sehr unterschiedlichen Todesursachen der in die Studie eingeschlossenen Probanden. Schon die Verwendung des Begriffs „Redox-modulierende Substanzen“ anstelle von „Antioxidanzien“ zeigt hierbei den Wandel im Verständnis der komplexen Funktionen dieser chemisch sehr heterogenen Substanzgruppe.

Zunehmende Bedeutung erlangen in dieser Hinsicht pflanzliche Phenole, wie sie in vielen Lebensmitteln, Früchten, Kräutern und Gewürzen etc. in komplexer Zusammensetzung enthalten sind.

Das komplexe Netzwerk der im menschlichen Organismus als RedoxModulatoren metabolisch aktiven Mikronährstoffe, die man auf mehrere zehntausend beziffern muss, eröffnet für die Zukunft ein weites Betätigungsfeld für intelligent geplante wissenschaftliche Studien, lässt noch manche Überraschung erwarten und liefert eine Grundlage für innovative Produkte.

Prof. Dr. Dr. Karlheinz Schmidt 